\title{
The relationship between pharmacokinetic parameters of carbamazepine and therapeutic response in epileptic patients
}

\author{
Chahra Chbili'1 Anis Hassine², Aicha Laouani ${ }^{1}$, Sana Ben Amor ${ }^{2}$, Manel Nouira ${ }^{1}$, Sofiène Ben Ammou², \\ Saad Saguem ${ }^{1}$
}

\author{
${ }^{1}$ Metabolic Biophysics, Professional and Applied Toxicology Environmental Laboratory, \\ Department of Biophysics, Faculty of Medicine Sousse, Sousse University, Sousse, \\ Tunisia \\ ${ }^{2}$ Neurology Department of Central Hospital University (CHU), Sousse University, \\ Sousse, Tunisia
}

Submitted: 9 January 2015

Accepted: 24 April 2015

Arch Med Sci 2017; 13, 2: 353-360

DOI: https://doi.org/10.5114/aoms.2016.60090

Copyright @ 2016 Termedia \& Banach

\section{Abstract}

Introduction: The prescribed dose and carbamazepine plasma concentration to achieve the optimal therapeutic efficacy are highly variable from one patient to the other. Our study aimed to determine whether biological parameters may be used as plasma markers that can individually adjust the carbamazepine dose necessary to optimize therapeutic efficacy.

Material and methods: Ninety-four epileptic patients under carbamazepine monotherapy and who have never used combination therapy were recruited from the consecutive admissions at the Department of Neurology "CHU Sahloul" of Sousse Central Hospital in Tunisia from February 2010 to April 2011. The patients were monitored for epilepsy for three years on average. Carbamazepine and 10,11-epoxide-carbamazepine concentrations were analyzed through high-performance liquid chromatography. Simultaneously, therapeutic efficacy was assessed through the annual number of seizures in each patient. Results: Our results showed the absence of any significant correlations between specific dose $(\mathrm{mg} / \mathrm{kg} /$ day), carbamazepine plasma concentrations and therapeutic efficacy $(r=0.0025, p=0.30 ; r=0.1584, p=0.38$ respectively), whereas both plasma 10,11-epoxide-carbamazepine concentration and 10,11-epoxide-carbamazepine to plasma carbamazepine ratio were closely correlated with therapeutic efficacy $(r=0.34, p=0.03 ; r=0.45$, $p=0.008$ respectively). The optimum therapeutic response was observed among patients who simultaneously had a plasma concentration of $0.8 \mu \mathrm{g} /$ $\mathrm{ml}$ of metabolite and $5.5 \mu \mathrm{g} / \mathrm{ml}$ of carbamazepine.

Conclusions: The results suggest that plasma levels of both carbamazepine and of 10,11-epoxide-carbamazepine must be set to achieve an optimum therapeutic response.

Key words: carbamazepine, 10,11-epoxide-carbamazepine, number of seizures, epileptic patients.

\section{Introduction}

Carbamazepine (CBZ) is one of the most widely prescribed antiepileptic drugs for the treatment of partial and generalized tonic-clonic seizures $[1,2]$. It can be used alone or in association with other drugs in the treatment of epilepsy [3-6].

\author{
Corresponding author: \\ Chachra Chbili PhD/ \\ Saad Saguem \\ Faculty of Medicine Sousse \\ Street Mohamed El Karoui \\ 4000 Sousse, Tunisia \\ Phone: +21693645001 \\ E-mail: chahra.chbili@ \\ yahoo.com, \\ khaled_saguem@yahoo.fr
}


The main metabolic pathway is catalyzed by CYP3A4, which is the most important cytochrome P450 enzyme that is intensely expressed in the liver $[1,7]$. Its major metabolite is an active epoxide: carbamazepine-10,11-epoxide (CBZ-E) [8-10]. The metabolite is fully converted by epoxide-hydrolase (EPHX1) to carbamazepine-10,11-diol, which is excreted in the urine in a free or a conjugated form [11]. These enzymes may constitute modulating factors of metabolic profiles of CBZ and can be implicated in the control of the therapeutic efficacy and toxicity of the CBZ [12-15].

In practice, therapeutic efficacy of CBZ is obtained with varying dosages. For some patients, "seizure free" is ensured by low doses. For others, even high doses may be insufficient. The therapeutic efficacy of CBZ is not easily controlled due to the difficulty to unify the prescribed dose and/ or necessary plasma concentration of CBZ to improve therapeutic efficacy. Indeed, the prescribed dose and the plasma concentration of CBZ that are necessary to achieve the effective therapeutic dose are highly variable from one patient to another in epileptic patients. In this study, we proposed to determine pharmacokinetic parameters that could be used as plasma markers that may individually adjust the dose of CBZ necessary to optimize the efficiency of therapy.

\section{Material and methods}

\section{Subjects}

Ninety-four patients with epileptic disease (34 patients with symptomatic epilepsy disease and 60 patients with cryptogenic epilepsy disease) were recruited for this study. These patients were composed of 40 men and 54 women with a mean age of 38.26 years. The ages ranged from 14 to 80 years, and the patients were recruited from the Neurology Department of Central Hospital University (CHU), Sousse, Tunisia for 14 months between February 2010 and April 2011. Most of the patients suffered from focal seizures with or without secondary generalization (50 patients, $53.19 \%$ ), whereas the rest of the patients suffered from generalized tonic-clonic seizures (44 patients, $46.80 \%$ ).

Table I. Clinical characteristics of epileptic patients

\begin{tabular}{|lc|}
\hline Total number of patients studied & 94 \\
\hline Seizure type (partial/generalized) & $50 / 44$ \\
\hline $\begin{array}{l}\text { Epilepsy type } N(\%) \\
\text { (symptomatic/cryptogenic) }\end{array}$ & $34 / 60$ \\
\hline Sex (M/F) & $40 / 54$ \\
\hline Age [years] & $38.26 \pm 17$ \\
\hline Weight [kg] & $63.3 \pm 7.6$ \\
\hline CBZ daily dose [mg/kg/day] & $8.18 \pm 3.18$ \\
\hline CBZ plasma concentration $[\mu g / m l]$ & $6.18 \pm 2.78$ \\
\hline
\end{tabular}

This study was approved by the local ethical committee. Informed consent was obtained from each study participant. The physical characteristics of the patients are given in Table I.

All the patients in this study were chronically treated with CBZ, and they did not take any other associated treatment to obtain optimal autoinduction by CBZ and to consider the steady state achieved in all patients. Patients were on carbamazepine for 8.16 months on average (range: 1-36 months). They were monitored for epilepsy for three years on average. Periodic clinical monitoring was performed every three months. As recommended, we performed a complete blood count and liver function test before initiation of treatment. These two tests were performed once a week for the first month of treatment and periodically thereafter [16]. No significant abnormalities were noted in hematological analysis. There were histories of stroke in 12 cases (receiving acetylsalicylic acid 125 to $250 \mathrm{mg} /$ day or DL-lysine acetylsalicylate 100 to $250 \mathrm{mg} /$ day). Intravenous alteplase was not administered in any case because of the delay in consulting or the contraindications. Also, there was trauma in 5 cases (without any medication). In trauma patients and in the case of stroke, the first seizure had occurred at least 10 months later. Patients had not suffered from sequelar headache. Prescribing analgesics was not justified. Cerebral magnetic resonance imaging (MRI) showed malformations of cortical development and tumor in 10 and 3 cases, respectively. Moreover, during our study, no neurological adverse effects (ataxia, diplopia) were observed. However, two major side effects were observed: skin lesions in 2 patients and benign digestive disorders in 11 cases. Ten patients suffered from a transient increase in gamma GT.

\section{Method}

All patients systematically had one blood sample taken before inclusion for analysis of CBZ plasma concentration. We included only patients with two closely spaced values. The samples were taken at 8:00 am before the morning dose. The plasma samples were analyzed with high-performance liquid chromatography in the Metabolic Biophysics, Professional Toxicology, and Applied Environmental Laboratory, Department of Biophysics, Medicine Faculty of Sousse, Sousse, Tunisia. The determination of the plasma levels of CBZ and CBZ-E in human plasma was conducted using previously described high-performance liquid chromatography (HPLC)-based assays [17].

\section{Statistical analysis}

The Pearson correlation statistical test was used to study the possible relationship between pharma- 
cokinetic parameters such as plasma concentrations of CBZ and CBZ-E, metabolic ratio of CBZ-E/CBZ, and the annual number of seizures in each patient. Fitted curves were obtained through Origin 6.0.

\section{Results}

Periodic clinical monitoring allowed the monitoring of the treatment efficacy through the number of seizures recorded annually in each patient, and it was also used as the criterion for assessing the therapeutic efficacy as recommended by the International League Against Epilepsy (ILAE) [18].

\section{Relationship between daily dosage and annual number of seizures}

One of the parameters pertaining to the specific dosage $(\mathrm{mg} / \mathrm{kg} /$ day) of CBZ was assessed. Considering only patients who developed seizures, the specific dosage as shown in Figure 1 was not significantly correlated with the annual number of crises $(r=0.0025, p=0.30)$.

Furthermore, the mean carbamazepine specific doses in patients who did not develop seizures and in patients who developed seizures were evaluated

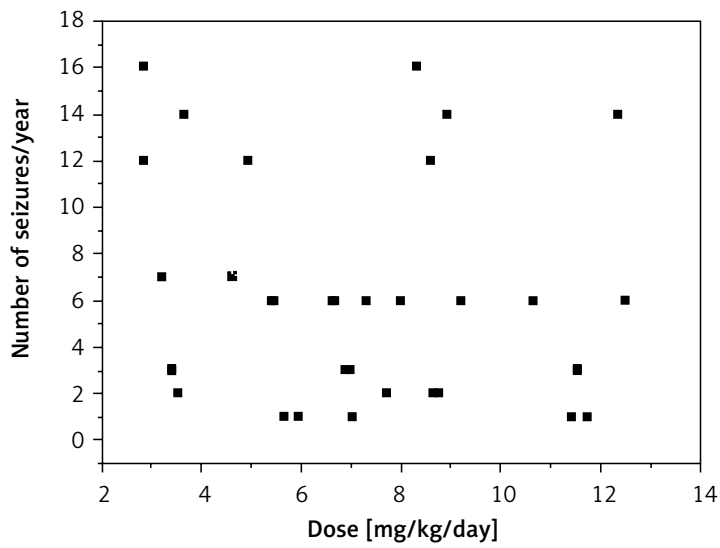

Figure 1. Variability of annual numer of seizures depending on the dosage

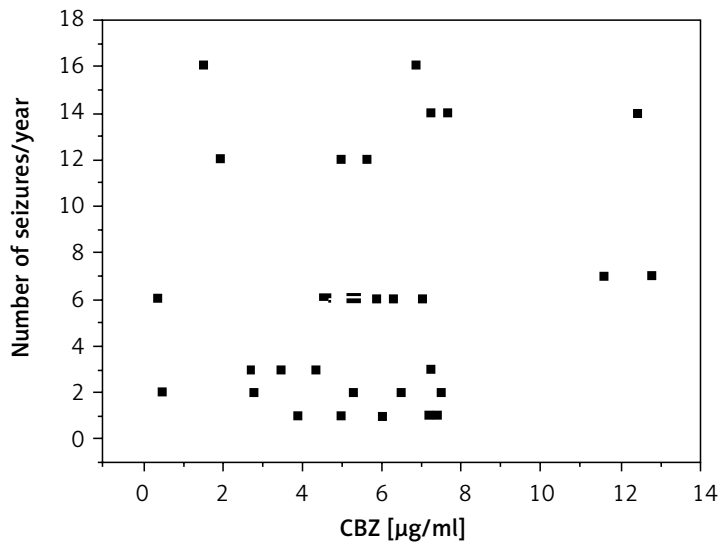

Figure 3. Variability of the annual number of seizures depending on the carbamazepine plasma concentration as $8.06 \pm 3.03 \mathrm{mg} / \mathrm{kg} / \mathrm{day}$ and $8.55 \pm 3.78 \mathrm{mg} / \mathrm{kg} / \mathrm{day}$, respectively. The recorded difference was not statistically significant $(p=0.08)$ (Figure 2 ). It thus appears that the specific dose of CBZ is not a parameter for predicting therapeutic efficacy as assessed by the number of annual seizures that occurred. Choosing this dosage depends on variable empirical data that vary from one patient to another.

\section{Relationship between carbamazepine plasma concentration and annual number of seizure}

To study the role of carbamazepine plasma concentration as a parameter that could potentially predict the therapeutic efficacy of CBZ in epileptic patients, we correlated this with the annual number of seizures that occurred. Figure 3 shows the total absence of such a correlation $(r=0.1584, p=0.38)$.

A comparison of mean values of carbamazepine plasma concentration in patients with and without seizures $(6.74 \pm 2.52 \mu \mathrm{g} / \mathrm{ml}$ and 5.69 $\pm 2.94 \mu \mathrm{g} / \mathrm{ml}$, respectively) showed a non-significant difference ( $p=0.07$ ) (Figure 4$)$. Our results thus show that the carbamazepine plasma con-

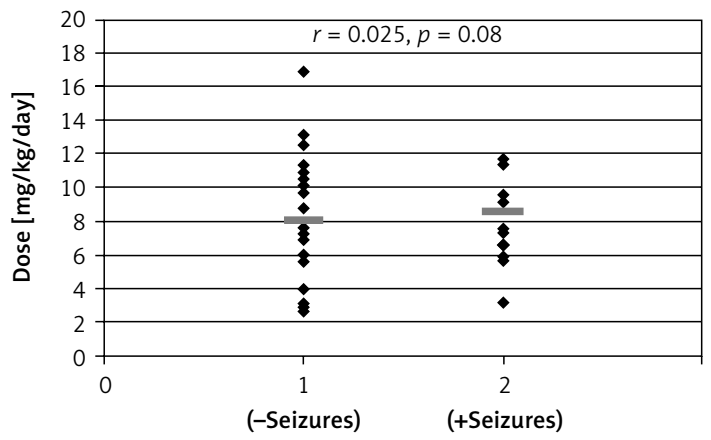

Figure 2. Variability in mean dose per $\mathrm{kg}$ of CBZ. Group 1: patients without seizures (-seizures) and group 2: patients with seizures (+seizures)

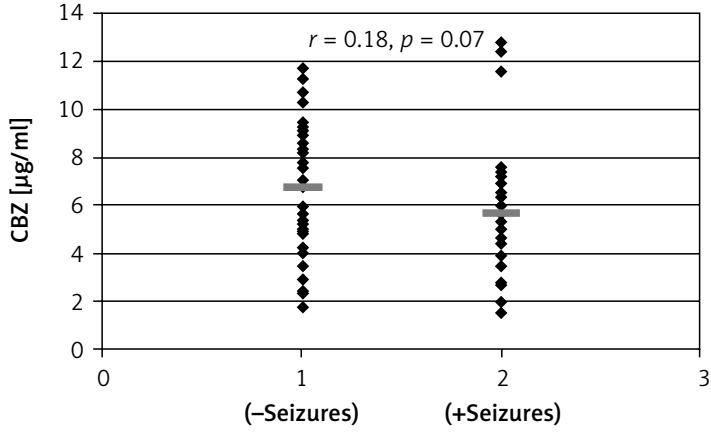

Figure 4. Variability of mean carbamazepine plasma concentrations in patients of group 1: without seizures (-seizures) and in patients of group 2: with seizures (+seizures) 
centration does not allow an individual scale to predict therapeutic response.

\section{Relationship between 10,11-epoxide- carbamazepine plasma concentration and annual number of seizures}

In contrast to the carbamazepine plasma concentration, the plasma concentration of CBZ-E was found to be significantly correlated with the annual number of seizures ( $r=0.34, p=0.03)$ using polynomial regression (Figure 5 ).

According to these results, the optimal therapeutic efficacy corresponding to the minimum number of seizures was recorded when the plasma concentration of CBZ-E was around $0.8 \mu \mathrm{g} / \mathrm{ml}$. It thus appears that the plasma concentration of CBZ-E could be a marker of therapeutic response. Taking into account that CBZ-E appears as a more suitable marker than CBZ in predicting the therapeutic efficacy, it could be at least partially due to its more polar character than CBZ, and therefore it is less bound to plasma proteins.

\section{Relationship between metabolic ratio CBZ-E/CBZ and annual number of seizures}

One of the parameters that could influence the therapeutic efficacy is one related to the enzymatic activity of CYP3A4 responsible for the bulk of the metabolic pathways of CBZ. The metabolic index reflecting such activity can be represented by the ratio of CBZ-E/CBZ. Figure 6 shows that it is well correlated with the number of annual seizures $(r=0.45, p=0.008)$ using polynomial regression.

The optimal efficiency obtained for a value of the metabolic ratio of CBZ-E/CBZ is around $15 \%$.

\section{Discussion}

The therapeutic efficacy in epileptic patients chronically treated with CBZ was evaluated

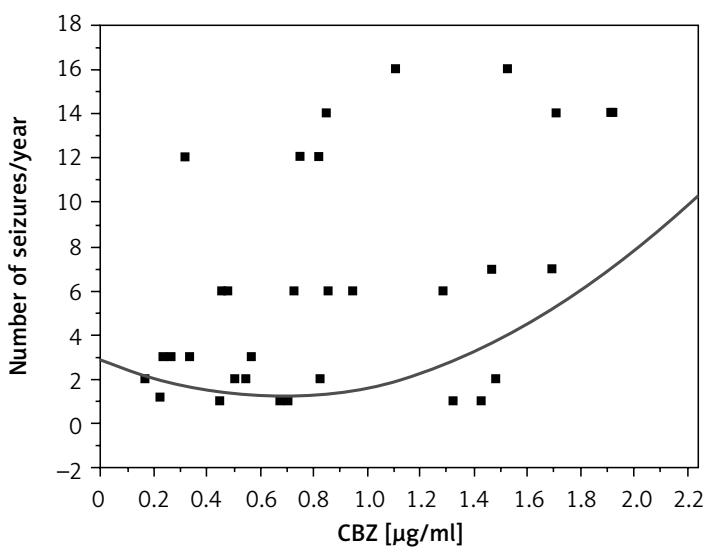

Figure 5. Variability of the annual number of seizures depending on the CBZ-E plasma concentration through the number of annual seizures that occurred in each patient. This therapeutic efficacy may depend on several factors. The pharmacokinetic factor could be one of the parameters likely to intervene in the interindividual variability.

This study aimed to examine the impact of metabolic factors such as plasma concentrations of CBZ and CBZ-E and the metabolic ratio CBZ-E/ $C B Z$ on the clinical response evaluated as the number of annual seizures occurring in each epileptic patient.

In this regard, our data failed to show a significant correlation between CBZ dosage and measurement of plasma CBZ concentration and the therapeutic effects evaluated by the annual number of seizures. On the other hand, our results are in partial agreement with the published data [19]. Johannessen et al. confirmed that in case of long-term treatment, important interindividual differences in plasma protein binding may be observed [19]. It could be an important determinant of drug efficacy.

Our results showed a significant association between plasma 10,11-epoxide-carbamazepine concentration and clinical response to treatment as evaluated by the annual numbers of seizures. This result is consistent with those obtained by several authors, in which the importance of the contribution of the active metabolite CBZ-E to therapeutic efficacy was reported [20-22]. Moreover, a more significant correlation was also found between CBZ-E to CBZ metabolic ratio and the response to treatment as evaluated by the annual numbers of seizures. This result is consistent with the results obtained by Fagiolino et al., which showed that to achieve a good response, the ratio should be between $12 \%$ and $18 \%$ [8].

Thus, our results suggest that plasma 10,11-epoxide-carbamazepine level and CBZ-E-to-CBZ ratio must be simultaneously considered to predict the therapeutic effect. The simultaneous increases of

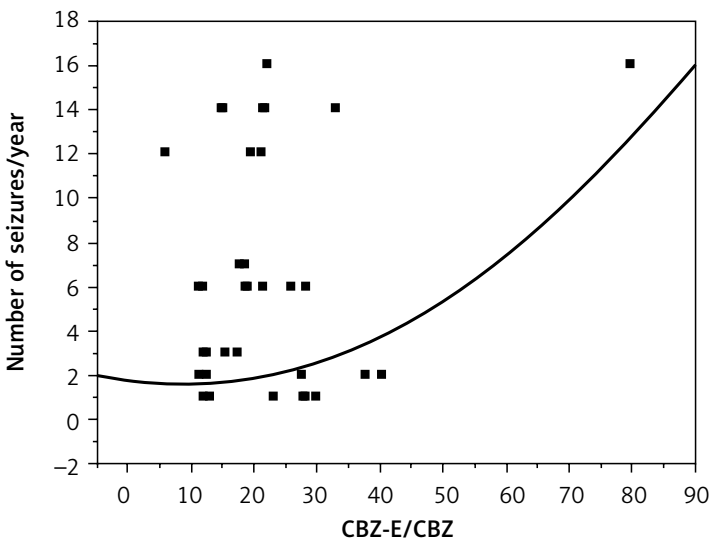

Figure 6. Variability of the annual number of seizures depending on the metaboli ratio CBZ-E/CBZ 
both plasma 10,11-epoxide-carbamazepine level and CBZ-E-to-CBZ ratio appear as a factor that significantly contributes to the increase of the therapeutic efficacy of CBZ in epileptic patients.

By simultaneously considering the two conditions found necessary to achieve a better therapeutic efficacy, plasma carbamazepine concentration should take into account the values of plasma concentration of CBZ-E $(0.8 \mu \mathrm{g} / \mathrm{ml})$ and of the metabolic ratio CBZ-E/CBZ (0.15), and it should thus be around $5.5 \mu \mathrm{g} / \mathrm{ml}$.

A carbamazepine plasma concentration of $5.5 \mu \mathrm{g} / \mathrm{ml}$, and plasma concentration of CBZ-E of $0.8 \mu \mathrm{g} / \mathrm{ml}$ constitute the values that must be achieved in epilepsy patients treated with CBZ, regardless of the patient considered. To the best of our knowledge, this is the first study to show that both parameters must be simultaneously set to achieve the optimum efficacy of CBZ.

It thus appears that the therapeutic efficacy of CBZ evaluated through the number of seizures that was recorded depends not only on the plasma carbamazepine concentration but also on the metabolite concentration of CBZ-E. Our results show that the plasma carbamazepine and CBZ-E concentrations should be simultaneously set to the respective values of $5.5 \mathrm{mg} / \mathrm{ml}(r=0.1584, p=$ $0.38)$ and $0.8 \mathrm{mg} / \mathrm{ml}(r=0.34, p=0.03)$ to achieve the optimal therapeutic effect in all patients.

In a previous study, a similar result was found in a group of patients with bipolar disorder who were treated with CBZ [17]. As evaluated by the Brief Psychiatric Rating Scale (BPRS) score, the optimal therapeutic efficacy was found when the plasma concentrations of CBZ and CBZ-E were simultaneously set at $7 \mu \mathrm{g} / \mathrm{ml}(r=0.28, p=0.19)$ and $1.4 \mu \mathrm{g} / \mathrm{ml}(r=0.78, p=0.0001)$, respectively [17]. In this case, the values obtained in the previous study are more meaningful than those obtained in our study. This difference between the two diseases could be explained by the instrument used to evaluate therapeutic efficacy. In the case of bipolar diseases, the authors used the BPRS score, which constitutes an objective and accurate parameter. However, in this study, the therapeutic efficacy was evaluated through the number of seizures over a relatively long period that was reported by the patient and/or his informants.

The differences found between each of the values to be set may reflect the relative difficulty in the assessment of therapeutic efficacy in epilepsy compared with a more accurate assessment of therapeutic efficacy in patients with bipolar disorder. Based on these results, the parameter values to be set found in the case of epilepsy are less precise, and therefore it would be better to consider intervals of values for each parameter.

Thus, after at least one month of CBZ chronic treatment, CBZ-E plasma concentration should be maintained below $0.8 \mu \mathrm{g} / \mathrm{ml}$. Considering that CBZ-E/CBZ pre-dose plasma concentration ratios are normally between $0.10 \mu \mathrm{g} / \mathrm{ml}$ and $0.40 \mu \mathrm{g} / \mathrm{ml}$, an updated therapeutic range for CBZ plasma levels might be proposed as from $2 \mu \mathrm{g} / \mathrm{ml}$ to $8 \mu \mathrm{g} / \mathrm{ml}$. To achieve the previous pharmacokinetic conditions necessary for obtaining the optimal therapeutic efficacy in epileptic patients treated with CBZ, we proposed a methodology using a protocol with several successive stages whose final objective would be to individually set the carbamazepine and the metabolite CBZ-E plasma concentrations to the values found previously. The daily dose of carbamazepine administered to the patient would be progressively increased until a carbamazepine plasma concentration that is equal to $5.5 \mu \mathrm{g} / \mathrm{ml}$ is obtained. The determination of the plasma concentration of CBZ-E is then achieved by HPLC according to the proposed method. We can then modulate the plasma concentration of CBZ-E found through the action of epoxide hydrolase, which is involved in the biotransformation of CBZ-E into carbamazepine-10,11-diol [23]. It comes to associate effectors of the epoxide hydrolase. This should be an inhibitor drug such as valproic acid when the plasma concentration of CBZ-E is lower than sought $(0.8 \mu \mathrm{g} / \mathrm{ml})$. On the other hand, it should be an inducer drug of epoxide hydrolase such as phenobarbital when the plasma concentration of CBZ-E is higher than the desired value.

It is important to mention that the treatment of epilepsy with CBZ is sometimes associated with valproic acid (VPA) or phenobarbital (PB). It has been reported that such an association leads to an improvement in the therapeutic efficacy of CBZ [24]. Thus, two anti-epileptic drugs may have antagonistic actions as they may expose the patient to additive side effects $[6,25]$. The ideal combination must demonstrate a pharmacological synergy defined by an improvement in the efficiency with similar toxicity, similar efficacy with reduced toxicity, or ideally improved efficiency with reduced toxicity [26]. It is guaranteed, especially, if the mechanism of action is different [27]. Thus, carbamazepine, phenobarbital, and phenytoin (PHT) are potent enzyme inducers as they are able to increase the level of cytochrome P450 activity. Concomitant inducer drugs may increase metabolism of the co-administered drug and possibly cause a loss of therapeutic efficacy. The extent of enzyme induction is dose-dependent and could be beneficial, especially if these drugs induce the same P450 isoenzymes. When PB, PHT, and CBZ are co-administered with either VPA, lamotrigine (LTG), topiramate (TPM), or tiagabine (TGB), they induce an increase in the metabolism of these drugs and a subsequent reduction in their halflives. For example, the plasma concentration of 
VPA was reduced by $76 \%$ when co-administered with $\mathrm{PB}$, by $49 \%$ with $\mathrm{PHT}$, and by $66 \%$ with $\mathrm{CBZ}$ [28]. It may be necessary to increase the dosage of anti-epileptic drugs (AED) assigned to maintain clinical efficacy of the combination therapy. In some patients with epilepsy and psychiatric disorders (e.g., depression), it may be necessary to co-administer psychotropic drugs and AED. Drug interactions between these two classes of agents focus on cytochrome P450 enzymes. For example, the enzyme-inducing $A E D P B, P H T$, and $C B Z$ raise metabolism of tricyclic antidepressants (TCAs) including nortriptyline, imipramine, nomifensine, and trazodone; and tricyclic antidepressants inhibit the metabolism of some anti-epileptic drugs [29]. The result is a reduction in the plasma concentration of tricyclic antidepressants, with an increase in plasma concentrations of co-administered AED. For example, fluoxetine (Prozac) inhibits the enzymes CYP3A4, CYP2D6, and CYP2C19, which may result in an increase in the plasma concentrations of CBZ and PHT. The monitoring of plasma concentrations of these drugs may be useful in preventing the adverse consequences of drug interactions [29]. Various factors must be considered during treatment, and it is important to try to identify people who may be sensitive to drug interactions before treatment is initiated. It is difficult to predict the extent of drug interactions due to the large interindividual variation between patients. This interindividual variation could be related to genetic polymorphism of isoenzymes involved in drug metabolism, particularly the enzyme CYP3A4, which is responsible for the conversion of CBZ in several metabolites. The most important one is 10,11-epoxy-carbamazepine (CBZ-E) [10].

Another catabolic enzyme of CBZ is microsomal epoxide hydrolase, which catalyzes the hydrolysis reaction of CBZ-E into 10,11-dihydroxy-10,11-dihydrocarbamazepine (DiOH-CBZ) [3, 30]. The genes encoding CBZ-metabolizing enzymes are highly polymorphic. Over 40 single nucleotide polymorphisms (SNPS) in the CYP3A4 gene have been reported (http://www.imm.ki.se/CYPalleles). There are particularly certain allelic variants of CYP3A4, such as CYP3A4*2, *4, *5, *6, * 17 , and *18, which cause a reduction or an increase in enzyme activity [12, 13].

The SNP CYP3A4*1G g.20230G >A (rs2242480) is common in the Chinese population, with a high allele frequency of about $26.9 \%$ [31-33]. Several studies have demonstrated that the CYP3A4*1G variant affects metabolism of CYP3A4 substrates in vitro and in vivo [14, 34-37], indicating that the CYP $3 A 4^{\star} 1 G$ polymorphism may affect CYP3A4 activity. Also, recent studies demonstrated that the CYP3A4*1B variant can influence drug metabolism [38]. The EPHX1 gene encoding the micro- somal epoxide hydrolase ( $\mathrm{mEH}$ ) enzyme is also highly polymorphic. The two common variants c.337T>C (rs1051740) and c.416A>G (rs2234922) have been demonstrated to change the catalytic activity of $\mathrm{mEH}$ both in vitro and in vivo [39-42]. Patients with the variant c.337T>C or c.416A>G allele tended to have higher CBZ doses or lower CBZ concentration-dose ratios (CDRs) $[30,43]$. Moreover, according to the literature, EPHX1 c.337T>C polymorphisms may contribute to the risk of cutaneous disorders [15].

Although this study did not focus on combination therapy in epileptic patients, if the co-administration of two drugs is necessary, the choice of the inhibitor or inducer drug should be guided by its action not only on CYP450 but especially on epoxide hydrolase. These considerations will be important if we intend to adjust the metabolic ratio.

Pharmacokinetics can lead to screening tests that would allow physicians to select the most appropriate treatment combinations for individual patients based on their genetic profiles. Doctors should be able to make more rational decisions about combination therapy with the maximum therapeutic benefit and minimal toxicity.

In conclusion, these preliminary results show that plasma CBZ-E and CBZ-E-to-CBZ ratio were successfully used to predict therapeutic efficacy among epileptic patients treated with CBZ. Our results show an interesting proof that $C B Z-E$ has a risk bénéfit on chronic treatment of $C B Z$ indicating the need to monitor plasma concentrations of CBZ-E. Thus, a new therapeutic strategy based on the simultaneous assessments of plasma CBZ and CBZ-E concentrations among epileptic patients treated with CBZ was proposed to adjust these parameters at values leading to the optimum efficacy.

However, due to the limited number of patients recruited in this study, these results should be viewed with caution, and more data are needed before any definitive conclusion can be drawn.

\section{Acknowledgments}

This study was supported by the "Higher Education and Scientific Research Ministry" in Tunisia. We thank all the staff of the Laboratory of Metabolic Biophysics, Professional and Applied Environmental Toxicology for their help and assistance.

\section{Conflict of interest}

The authors declare no conflict of interest.

\section{References}

1. Pellock JM. Treatment considerations: traditional antiepileptic drugs. Epilepsy Behav 2002; 3 (6S1): 18-23. 
2. Ahmed SN, Siddiqi ZA. Antiepileptic drugs and liver disease. Seizure 2006; 15: 156-64.

3. Liu H, Delgado MR. The influence of polytherapy on the relationships between serum carbamazepine and its metabolites in epileptic children. Epilepsy Research 1994; 17: 257-69.

4. Bernus I, Dickinson RG, Hooper WD, Eadie MJ. The mechanism of the carbamazepine-valproate interaction in humans. Br J Clin Pharmacol 1997; 44: 21-7.

5. Patsalos PN, Fröscher W, Pisani F, van Rijn CM. The importance of drug interactions in epilepsy therapy. Epilepsia 2002; 43: 365-85.

6. Chmielewska B, Lis K, Rejdak K, Balcerzak M, Steinborn B. Pattern of adverse events of antiepileptic drugs: results of the a ESCAPE study in Poland. Arch Med Sci 2013; 9: 858-64.

7. Yuki H, Honma T, Hata M, Hoshino T. Prediction of sites of metabolism in a substrate molecule, instanced by carbamazepine oxidation by CYP3A4. Bioorg Med Chem 2012; 20: 775-83.

8. Fagiolino P, Vázquez M, Olano L, Delfino A. Systemic and presystemic conversion of carbamazepine to carbamazepine-10,11-epoxide during long term treatment. J Epilepsy Clin Neurophysiol 2006; 12: 13-6.

9. Breton H, Cociglio M, Bressolle F, Peyriere H, Blayac JP, Hillaire-Buys D. Liquid chromatography-electrospray mass spectrometry determination of carbamazepine, oxcarbazepine and eight of their metabolites in human plasma. J Chromatogr B Analyt Technol Biomed Life Sci 2005; 828: 80-90.

10. Bu HZ, Kang P, Deese AJ, Zhao P, Pool WF. Human in vitro glutathionyl and protein adducts of carbamazepine-10,11-epoxide, a stable and pharmacologically active metabolite of carbamazepine. Drug Metab Dispos 2005; 33: 1920-4.

11. Aldaz A, Ferriols R, Aumente D, et al. Pharmacokinetic monitoring of antiepileptic drugs. Farm Hosp 2011; 35 326-39.

12. Lee SJ, Goldstein JA. Functionally defective or altered CYP3A4 and CYP3A5 single nucleotide polymorphisms and their detection with genotyping tests. Pharmacogenomics 2005; 6: 357-71.

13. Dally H, Edler L, Jager B, et al. The cyp3a4*1b allele increases risk for small cell lung cancer: effect of gender and smoking dose. Pharmacogenetics 2003; 13 607-18.

14. Dong ZL, Li H, Chen QX, et al. Effect of CYP3A4*1G on the fentanyl consumption for intravenous patient-controlled analgesia after total abdominal hysterectomy in Chinese Han population. J Clin Pharm Ther 2012; 37: 153-6.

15. He XJ, Jian $L Y, H e X L$, et al. Association of $A B C B 1, C Y$ P3A4, EPHX1, FAS, SCN1A, MICA, and BAG6 polymorphisms with the risk of carbamazepine-induced Stevens-Johnson syndrome/toxic epidermal necrolysis in Chinese Han patients with epilepsy. Epilepsia 2014; 55 1301-6.

16. Lévy-Chavagnat D. Stratégies de traitement des syndromes épileptiques. Actualités Pharmaceutiques 2008; 475: 10-6.

17. Chbili C, Bannour S, Khlifi S, Ben Hadj Ali B, Saguem S. Relationships between pharmacokinetic parameters of carbamazepine and therapeutic response in patients with bipolar disease. Ann Biol Clin (Paris) 2014; 72: 453-9.

18. Kwan P, Arzimanoglou A, Berg AT, et al. Definition of drug resistant epilepsy: consensus proposal by the ad hoc Task Force of the ILAE Commission on Therapeutic Strategies. Epilepsia 2010; 51: 1069-77.
19. Johannessen SI, Gerna M, Bakke J, Strandjord RE, Morselli PL. CSF concentrations and serum protein binding of carbamazepine and carbamazepine-10,11-epoxide in epileptic patients. Br J Clin Pharmacol 1976; 3: 575-82.

20. Potter JM, Donnelly A. Carbamazepine-10,11-epoxide in therapeutic drug monitoring. Ther Drug Monit 1998; 20: 652-7.

21. So EL, Ruggles KH, Cascino GD, Ahmann PA, Weatherford KW. Seizure exacerbation and status epilepticus related to carbamazepine-10,11-epoxide. Ann Neurol 1994; 35: 743-6.

22. Bourgeois BF, Wad N. Individual and combined antiepileptic and neurotoxic activity of carbamazepine and carbamazepine-10,11-epoxide in mice. J Pharmacol Exp Ther 1984; 231: 411-5.

23. Moreno J, Belmont A, Jaimes O, et al. Pharmacokinetic study of carbamazepine and its carbamazepine 10,11-epoxide metabolite in a group of female epileptic patients under chronic treatment. Arch Med Res 2004; 35: 168-71.

24. Mervyn J, Eadie MD. Place of antiepileptic drug combinations. J Clin Neurosci 1997; 4: 432-8.

25. Czuczwar SJ, Borowicz KK. Polytherapy in epilepsy: the experimental evidence. Epilepsy Res 2002; 52: 15-23.

26. Brodie MJ, Sills GJ. Combining antiepileptic drugs: rational polytherapy? Seizure 2011; 20: 369-75.

27. Stephen LJ, Forsyth M, Kelly K, Brodie MJ. Antiepileptic drug combinations: have newer agents altered clinical outcomes? Epilepsy Res 2012; 98: 194-8.

28. Perucca E, Tomson T. The pharmacological treatment of epilepsy in adults. Lancet Neurol 2011; 10: 446-56.

29. Monaco F, Cicolin A. Interactions between anticonvulsant and psychoactive drugs. Epilepsia 1999; 40: S71-6.

30. Hung CC, Chang WL, Ho JL, et al. Association of polymorphisms in EPHX1, UGT2B7, ABCB1, ABCC2, SCN1A and SCN2A genes with carbamazepine therapy optimization. Pharmacogenomics 2012; 13: 159-69.

31. Pisani F, Oteri G, Russo MF, et al. The efficacy of valproate lamotrigine comedication in refractory complex partial seizures: evidence for a pharmacodynamic interaction. Epilepsia 1999; 40: 1141-6.

32. Grimsley SR, Jann MW, Carter JG, et al. Increased carbamazepine plasma concentrations after fluoxetine coadministration. Clin Pharmacol Ther 1991; 50: 10-5.

33. Du J, Xing Q, Xu L, et al. Systematic screening for polymorphisms in the CYP3A4 gene in the Chinese population. Pharmacogenomics 2006; 7: 831-41.

34. Du J, Zhang A, Wang L, et al. Relationship between response to risperidone, plasma concentrations of risperidone and CYP3A4 polymorphisms in schizophrenia patients. J Psychopharmacol 2010; 24: 1115-20.

35. Zhang W, Chang YZ, Kan QC, et al. CYP3A4*1G genetic polymorphism influences CYP3A activity and response to fentanyl in Chinese gynecologic patients. Eur J Clin Pharmacol 2010; 66: 61-6.

36. Yuan R, Zhang X, Deng Q, Wu Y, Xiang G. Impact of CYP3A4* $1 \mathrm{G}$ polymorphism on metabolism of fentanyl in Chinese patients undergoing lower abdominal surgery. Clin Chim Acta 2011; 412: 755-60.

37. Miura M, Satoh S, Kagaya $H$, et al. Impact of the CYP $3 A 4^{*} 1 G$ polymorphism and its combination with CYP3A5genotypes on tacrolimus pharmacokinetics in renal transplant patients. Pharmacogenomics 2011; 12: 977-84.

38. Puranik YG, Birnbaum AK, Marino SE, et al. Association of carbamazepine major metabolism and transport pathway gene polymorphisms and pharmacokinetics 
in patients with epilepsy. Pharmacogenomics 2013; 14: 35-45.

39. Hassett C, Robinson KB, Beck NB, Omiecinski CJ. The human microsomal epoxide hydrolase gene (EPHX1): complete nucleotide sequence and structural characterization. Genomics 1994; 23: 433-42.

40. Hassett C, Lin J, Carty CL, Laurenzana EM, Omiecinski CJ. Human hepatic microsomal epoxide hydrolase: comparative analysis of polymorphic expression. Arch Biochem Biophys 1997; 337: 275-83.

41. Kitteringham NR, Davis C, Howard N, Pirmohamed M, Park BK. Interindividual and interspecies variation in hepatic microsomal epoxide hydrolase activity: studies with cisstilbene oxide, carbamazepine 10,11-epoxide and naphthalene. J Pharmacol Exp Ther 1996; 278 : 1018-27.

42. Nakajima Y, Saito Y, Shiseki K, et al. Haplotype structures of EPHX1 and their effects on the metabolism of carbamazepine-10,11-epoxide in Japanese epileptic patients. Eur J Clin Pharmacol 2005; 61: 25-34.

43. Makmor-Bakry M, Sills GJ, Hitiris N, Butler E, Wilson EA, Brodie MJ. Genetic variants in microsomal epoxide hydrolase influence carbamazepine dosing. Clin Neuropharmacol 2009; 32: 205-12. 